\title{
Determinants Of Multidrug Resistant Tuberculosis Among Adultsundergoing Treatment For Tuberculosis In Tigray Region, Ethiopia: A Case Control Study
}

Kidane Zereabruk ( $\sim$ zereabrukkidane@gmail.com )

Aksum University

Tensay Kahsay

Mekelle University

Hiyab Teklemichael

Mekelle University

Woldu Aberhe

Aksum University

Abrha Hailay

Aksum University

Guesh Mebrahtom

Aksum University

Gebrewahd Bezabih

Aksum University

Research article

Keywords: Determinants, Ethiopia, MDR TB, Tigray

Posted Date: December 12th, 2019

DOI: https://doi.org/10.21203/rs.2.15772/v2

License: (c) (i) This work is licensed under a Creative Commons Attribution 4.0 International License. Read Full License 


\section{Abstract}

Background Multidrug-resistant tuberculosis is a type of tuberculosis that is resistant to at least the first line anti-tuberculosis drugs namely, Rifampicin and Isoniazid. Multidrug-resistant tuberculosis has continued to be a challenge for tuberculosis control globally. Globally 600,000 people were newly eligible for Multidrugresistant tuberculosis treatment with an estimated 240,000 deaths annually. There are few numbers of multi drug resistant tuberculosis studies in different regions of Ethiopia. However, most of these studies were restricted only to a single hospital and there is no published information regarding multi drug resistant tuberculosis in Tigray region. Therefore, the aim of this study was to assess the determinants of multidrug resistant tuberculosis among adults undergoing treatment for tuberculosis in Tigray region, Ethiopia.Methods A hospital based unmatched case control study was conducted from April to June, 2019. Simple random sampling method was used to select 85 cases and 169 controls. Primary data was collected by face-to-face interview and secondary data by reviewing patients' charts using pretested structured questionnaires. The data were entered and cleaned using Epi data manager then exported to SPSS for analysis. Binary Logistic regression model was used to test the association between independent and dependent variables. Model fitness was checked using Hosmer-Lemeshow goodness-of-fit and Variance inflation factor was used to assess multi collinearity between the independent variables. Variables at p-value less than 0.25 in bivariate analysis was entered into a multivariable analysis to identify the determinant factors of multi drug resistant tuberculosis. Finally, level of significance was declared at pvalue $<0.05$. Results A total of 254 participants with 85 cases and 169 controls were included in this study. Of the respondents, $62(36.7 \%)$ among the controls and 48 (56.5\%) among the cases were living in rural residence. Rural residence [AOR=2.54;95\% Cl=1.34,4.83], $\mathrm{HIV}[\mathrm{AOR}=4.5 ; 95 \% \mathrm{Cl}=1.4,14.2]$, relapse [AOR=3.86;95\% Cl;1.98,7.5], return after lost follow up [AOR=6.29;95\% 1.64,24.2], treatment failure $[A O R=5.87 ; \mathrm{Cl}=1.39,24.8]$ were among the determinants of Multi drug resistance tuberculosis.Conclusion Rural residence, HIV, relapse, return after lost follow up and treatment failure were the identified determinant factors of Multi drug resistance tuberculosis.

\section{Background}

Tuberculosis (TB) is a communicable disease which is caused by Mycobacterium tuberculosis[1]. MDR-TB is a kind of tuberculosis that is resistant to at least to the isoniazid and rifampicin [2]. When someone who has no history of first-line TB treatment develops MDR-TB, it is called primary MDR-TB. When inappropriate treatment leads to the selection of spontaneously resistant strains, the disease is called secondary MDR-TB [3]. Both primary and secondary types of MDR-TB have been described in Ethiopia [4].

Globally, MDR-TB has sustained to be a challenge for TB control $[5,6]$. According to the 2016, World Health Organization report more than 600,000 people were newly eligible for MDR-TB treatment worldwide[7]. Even though MDR-TB is a rising concern in Africa, it is largely under-reported [8,9]. According to 2010 WHO report the number of MDR-TB was largely increasing in Africa [2]. The prevalence of MDR TB differs among countries and regions while high prevalence has been observed in the developing world[5]. Sub-Sahara African countries represent fourteen percent of the global burden of new MDR-TB cases [8]. Seven countries including Ethiopia in Africa are listed among the 30 high MDR-TB burden countries in the world [10]. 
According to 2016 WHO report, Ethiopia was ranked $8^{\text {th }}$ out of 30 high MDR-TB burden countries in the world with a prevalence rate of $2.7 \%(1.5-4.0)$ in newly and $14.0 \%(3.6-25.0)$ in previously treated patients [11].

MDR-TB is a cause of death for more than 240,000 people annually [7]. Patients infected with multiple-drug resistant strains are less likely to become cured the disease[12] .The cure rate for MDR TB is poor ,ranging from $6 \%$ to $59 \%$ [13]. The management of MDR-TB with second-line anti TB drugs is long which is more than two years, complicated process, and has high adverse effects and making MDR-TB costly and difficult to manage than drug-susceptible tuberculosis[15,16]. The average price of drugs alone for treating the MDR-

TB patient is 50-200 times higher than for treating a drug-susceptible TB patient [17]. The treatment cost of MDR TB is $2000-20,000$ US\$ per patient but it is in the range of 100-1000US\$ for drug-susceptible TB patients [7].

Globally, prevention of new infections of Mycobacterium tuberculosis and their progression to tuberculosis (TB) disease is critical to reduce the burden of disease and death caused by TB, and to achieve the End TB Strategy targets set for 2030 and 2035[18].

There are limited numbers of MDR-TB studies in different regions of Ethiopia [19, 20, 21]. Therefore, the main aim of this study was to identify the determinant factors MDR TB among adults undergoing treatment for tuberculosis in Tigray region, Ethiopia.

\section{Methods}

\section{Duration and Area of the Study}

The study was conducted in Tigray public hospitals. Tigray is found in the northern part of Ethiopia. Tigray regional state has two comprehensive specialized hospitals, 15 general hospitals, 22 primary hospitals, and 223 health centers. From the 15 general hospitals seven hospitals namely Kahsay Abera, Suhul, Adwa, Adigrat, Mekelle, Lemlem Karl and Alamata are giving MDR TB treatment services. There were 118 registered MDR TB patients in the above seven hospitals of Tigray regional state. The study was conducted from April to June, 2019 in those seven multi drug-resistant tuberculosis treatment center hospitals of Tigray regional state.

\section{Study Design}

Hospital-based unmatched case-control study was conducted.

\section{Study Population}

\section{Sample Size Determination and Sampling Technique}

\section{Sample Size Determination}


The required sample size was determined by using the Epi-Info version 7.2.2.12 from the previous study conducted in Ethiopia. The estimated sample size was determined based on the following assumptions: confidence interval of $95 \%$ at the power of $80 \%$, with the ratio of $1: 2$ (case to control). AOR=2.4, $95 \%$ $\mathrm{Cl}=95 \%$, Power $=80 \%$, percent controls exposed $=16.9$, and percent of cases exposed for cases was taken 32.8 from a study conducted in eastern Shoa, Ethiopia [21].

Finally, the sample size was 242. By adding a 5\% non-response rate the total sample size was 254 (85 and 169 controls).

\section{Sampling Techniques and Procedures}

First, the sample size was allocated proportionally to the seven MDR TB treatment centers of Tigray according to the number of patients registered to take the treatment. Then a frame of MDR-TB patients enrolled in second line drug treatment at MDR treatment centers of Tigray was created using the medical registration number. Finally, a simple random sampling technique was used to select the MDR TB cases. MDR-TB patients enrolled to second line-drug at MDR treatment center hospitals of Tigray taken as cases and simple random sampling technique was used to include 85 study participants from the list of MDR TB patient's registration book. 169 Controls were also selected by a simple random sampling technique from the TB registration book in similar hospitals.

\section{Data Collection Tools and Procedures}

The primary data were collected by face-to-face interviews using pretested structured questionnaires, while secondary data were collected by reviewing participant's medical charts using checklists for the matching study participants. Seven trained BSc nurses were assigned to conduct an interview with the participants and review the corresponding participant records. Three senior BSc nurse supervisors were assigned to supervise the whole data collection process. The questioner contained socio-demographic related factors, behavioral related factors and clinical related factors. Throughout the data collection process, data collectors were used the $\mathrm{N}-95$ respiratory mask to prevent from acquiring the disease. The data collection period was from April to June 2019.

\section{Data Quality Assurance}

Data quality was ensured by giving two days of training for data collectors and supervisors and by providing supervision during the data collection period. First, the questioner was adapted from a published paper in English form [21], then translated into Tigrigna (local language) and back-translated into English to ensure its consistency. Each questionnaire was checked for completeness before leaving the study area. The questionnaire was pretested on $5 \%$ of other patients who did not participated in the study for completeness and appropriateness to the local context. Based on the findings the necessary amendments have done. Every questionnaire was checked by the principal investigator on the spot.

\section{Data Processing and Analysis}


The data was coded and entered into Epi data manager version 4.4.3.1 then exported to SPSS version 20 for further statistical analysis.

Descriptive statistics such as frequencies, percentages, median, inter-quartile range were computed. Finally, the report was summarized and presented using, texts, tables, and figures. Binary Logistic regression model was used to test the association between independent and dependent variables. All variables at $p$-value $<0.25$ in bivariable logistic regression were entered into multivariable logistic regression to determine the association between a set of independent variables with the dependent variable. The odds ratio was estimated at $95 \% \mathrm{Cl}$ to show the strength of an association and statistical significance was declared at $\mathrm{p}$ value $<0.05$. The model fitness was checked using Hosmer-Lemeshow goodness-of-fit the $p$-value which was $>0.05$ which was fitted (0.436). The variance inflation factor (VIF) was used to assess multicollinearity between the independent variables.

\section{Study Variables}

The dependent variable was MDRTB (yes or no). The Independent variables included the sociodemographic variables (age, sex, religion, socio economic status, educational status, occupational status, marital status, residence, family size, the number of rooms in the patient's household, and number of windows), clinical related variables (HIV status, history of contact with known TB patient, history of contact of with known TB patient, other underlying/chronic disease, number of TB episodes TB, outcome, history of interruption the first line anti TB, directed observed therapy, encountered side effects, category of TB, and duration of firs-line treatment)and behavioral related variables (prison status, alcohol consumption and cigarette smoking). Data on socio-demographic characteristics, behavioral characteristics and some the clinical characteristics were collected through face to face interviews. The remained clinical characteristics were collected by the review of the patient records and registration books.

\section{Results}

\section{Socio-demographic characteristics}

A total of 254 participants with 85 cases and 169 controls were included in this study with a $100 \%$ response rate. The median and inter-quartile range age of the cases was $\mathbf{3 0} \pm \mathbf{1 8}$. The median and inter-quartile range age of the controls was $35 \pm 19$. Of the participants, 55 (64.7\%) were males among the cases and $99(58.3 \%)$ were females among controls (Table 1).

Regarding educational status, around 34 (40\%) participants among the cases and 60 (35.5\%) participants among controls had no formal education, $26(30.6 \%)$ participants among cases and 55(32.5\%) participants among controls had finished their primary school, $14(16.5 \%)$ participants among cases and $34(20.1 \%)$ participants among controls were secondary school, 11(12.9\%) participants among cases and $20(11.8 \%)$ participants among controls were college and above.

Of the respondents, 62 (36.7 \%) among the controls and 48 (56.5\%) among the cases were living in rural residence (Figure 1). 


\section{Behavioral related factors}

More than three fourth, 67 (78.8\%) cases and 127 (75.1\%) controls did not drink any alcohol. Of the cases, 7 (8.2\%) and 17 (10.1\%) from controls were cigarette smokers (Table 2).

\section{Clinical related factors}

Of the respondents $29(34.1 \%)$ of cases (MDR TB patients) and $120(71 \%)$ of controls (TB patients) were new patients. Eight $(9.4 \%)$ and $14(16.5 \%)$ among cases were return after lost follow up and treatment failure respectively. From the participants, 34 (40\%) among the cases and 31 (18.3\%) among controls were relapse patients (Figure 2).

Of the cases, 56 (65.9\%) were faced with two and more episodes of tuberculosis and 120 (71\%) among controls were faced only one episode. Seventy (82.4\%), 163 (96.4\%) were non-reactive among cases and controls respectively. From the participants, 15 (17.6\%) among cases and $6(3.6 \%)$ among controls were HIV reactive patients (Table 3 )

\section{Determinants of Multidrug resistance Tuberculosis}

The bivariate logistic regression analysis showed that rural residence, TB episodes greater than or equal to two, treatment category (treatment after lost follow up, relapse and treatment failure), HIV and history of close contact with known TB were statistically associated with the development of MDR TB. However, after multivariable logistic regression analysis rural residence, HIV, relapse, lost follow up and treatment failure were significantly associated with MDR TB at $p$-value $<0.05$.

The odds of MDR TB among rural residence was 2.6 times as compared to urban residence [AOR=2.58; $95 \% \mathrm{Cl}=1.4-4.6]$. The odds of being MDR TB among HIV reactive participants was 4.4 times as compared to HIV non-reactive participants [AOR=4.4;95\% $\mathrm{Cl}=1.5-12.6]$. Treatment after lost follow up participants had 5.4 times high risk for MDR TB as compared to the newly diagnosed patients [AOR=5.4;95\% $\mathrm{Cl}=1.69-17$ ]. Relapse patients had 3.9 times high risk for the development of MDR TB as compared to new patients ( $A O R=3.86 ; 95 \% \mathrm{Cl}=1.98-7.5)$. Participants with first-line treatment failure patients were found 5 times more likely to develop MDR TB as compared to new patients [AOR=5.1;95\% Cl=2-13] (Table 4).

\section{Discussion}

In this study living in a rural setting, return after lost follow up, relapse, treatment failure, and HIV were found to be significantly associated with the occurrence of MDR-TB.

MDR-TB was significantly associated with residence area; living in a rural area increased the occurrence of MDR-TB compared with living in an urban area. This is similar to the studies conducted in east Shoa, Oromia region and Amhara regional state, Ethiopia $[23,24,25]$. This could be due to the huge proportion of people living rural area, difference in access to TB services, socio-economy and the level of knowledge about adherence to first-line anti TB treatment, as rural communities have poor adherence to treatment that likely leads to the MDR-TB [23].In contrast, a study conducted in southwestern Ethiopia showed that rural 
residence was not associated with the development of MDR TB[22]. This might be due to the sample size difference.

In this study, HIV was significantly associated with the occurrence of MDR-TB. This is supported by a study conducted in Oromia which found that HIV is a risk factor for the development of MDR-TB[24]. This might be due to drug mal-absorption in HIV infected patients since the patient is taking both ART medication and anti TB medications, especially ethambutol and rifampicin can lead to drug-resistance and treatment failure[26]. In contrast, HIV was not associated with the occurrence of MDR-TB in a study conducted in southwestern and, east Shoa, Ethiopia [22,23]. This discrepancy might be due to the sample size difference.

In this study, MDR TB was significantly associated with return after lost follow up (defaulting). This finding is similar with the study conducted in Brazil, Thailand, and Addis Ababa, Ethiopia [27-29]. This can be described by the increased exposure to anti-tuberculosis drugs. A study conducted in eleven countries has revealed that the elongated the time of exposure to anti-tuberculosis drugs, the more the chance for the development of MDR-TB [30]. This might be due to failure, deprived management of the patient, patient or clinician noncompliance, and absence of infection control practices in the health facilities [31,32].

This study revealed that relapse was significantly associated with the occurrence of MDR TB. This finding is supported by a study conducted in Brazil [29]. In the contrary, relapse was not associated with the occurrence of MDR-TB in a study conducted in Amhara regional state [25]. This difference might be due to the different in the selection of study participants and a relatively small proportion of relapse participants in a study conducted in Amhara regional state.

Treatment failure was significantly associated with the development of MDR-TB. This finding is similar to a study conducted in Jimma southwestern Ethiopia, Addis Ababa, Amhara regional state, Ethiopia [23, 27, 33]. The association between TB treatment failure and MDR-TB might be due to noncompliance by patients or health providers, lack of supervision of treatment, inappropriate drug regimens and inadequate drug supply that may increase the chance of drug resistance [23,34].

\section{Limitation Of The Study}

This study may have recall bias since some of the information such as interruption of anti TB medications, contact history with known TB and MDR TB were based on the previous recall of the study participants.

\section{Conclusion}

This study has identified rural residence, HIV, relapse, return after lost follow up and treatment failure as determinant factors for the occurrence of MDR TB.

\section{List Of Abbreviations}

AIDS: Acquired Immune Deficiency Syndrome; OR: Adjusted Odds Ratio; BCG: Bacille Calmette Guerin; Cl: Confidence interval; COR: Crude Odds Ratio; DM: Diabetes Mellitus; DST: Drug Susceptibility Testing; HIV: 
Human Immune Virus; MDRTB: Multi Drug Resistant Tuberculosis; RR: Rifampicin Resistant; SPSS:

Statistical Package for Social Science; TB: Tuberculosis; WHO: World Health Organization.

\section{Declarations}

\section{Ethics approval and consent from the participant}

Ethical clearance was obtained from Mekelle University College of health science institutional review board (IRB) (ERC 1283/2019). Furthermore, prior to conducting the study, the purpose and objective of the study were described to the study participants and written informed consent was obtained. Respondents were allowed to refuse or discontinue participation at any time they want. Confidentiality was assured and maintained throughout the study period.

\section{Competing interests}

The authors declare that they have no competing interests.

\section{Funding}

Not applicable

\section{Consent for publication}

Not applicable

\section{Availability of data and materials}

The datasets used and/or analyzed during the current study are available from the corresponding author on reasonable request.

\section{Author's contributions}

$\mathrm{KZ}$ was made significant contributions to the conception, design of the work, methodology, analysis, data interpretation and wrote the final manuscript. TK, HT and GB had equally contributed to the analysis and interpretation of the data. WA, AA and GM have made significant contributions in reviewing overall the study in analysis, interpretation of data, have drafted the manuscript and essentially revised the work. All authors read and approved the final manuscript.

\section{Acknowledgments}

We would like thank to Mekelle University, supervisors, data collectors and study subjects.

\section{References}

1. World Health Organization Global tuberculosis report, Geneva. 2018: World Health Organization. 
2. World Health Organization drug-resistant tuberculosis now at record levels. Geneva: World Health Organization, 2010.

3. Loddenkemper, R., D. Sagebiel, and A. Brendel, Strategies against multidrug-resistant tuberculosis. European respiratory journal, 2002. 20(36 suppl): p. 66s-77s.

4. Abebe, G., et al., Relatively low primary drug resistant tuberculosis in southwestern Ethiopia. BMC research notes, 2012. 5(1): p. 225.

5. World Health Organization global tuberculosis control. 2010: World Health Organization.

6. Wright, A. and M. Zignol, Anti-tuberculosis drug resistance in the world: fourth global report: the world health organization/international union against tuberculosis and lung disease (who/union) global project on anti-tuberculosis drug resistance surveillance, 2002-2007. 2008: World Health Organization.

7. World Health Organization Global tuberculosis report 2016.

8. Migliori, G.B., et al., Review of multidrug-resistant and extensively drug-resistant TB: global perspectives with a focus on sub-Saharan Africa. Tropical Medicine \& International Health, 2010. 15(9): p. 1052-1066.

9. Amor, Y.B., et al., Underreported threat of multidrug-resistant tuberculosis in Africa. Emerging infectious diseases, 2008. 14(9): p. 1345.

10. World Health Organization Global tuberculosis report,Geneva. 2017.

11. World Health Organization., Global tuberculosis report 2018. 2018.

12. Goble, M., et al., Treatment of 171 patients with pulmonary tuberculosis resistant to isoniazid and rifampin. New England journal of medicine, 1993. 328(8): p. 527-532.

13. Sharma, S. and A. Mohan, Multidrug-resistant tuberculosis. Indian Journal of Medical Research, 2004. 120: p. 354-376.

14. $\mathrm{MOH}$, Guidelines for clinical and programmatic management of TB, leprosy and TB/HIV in Ethiopia. 2012, Ministry of Health Addis Ababa.

15. Organization, W.H., The global MDR-TB \& XDR-TB response plan 2007-2008. 2007.

16. World Health Organization., Drug-resistant tuberculosis now at record levels. Geneva: World Health Organization, 2010.

17. Espinal, M.A., et al., Standard short-course chemotherapy for drug-resistant tuberculosis: treatment outcomes in 6 countries. Jama, 2000. 283(19): p. 2537-2545. Tuberculosis research and treatment, 2014. 2014.

18. World Health Organization (WHO), Global tuberculosis report. 2018.

19. Gobena, D., et al., Predictor of multidrug resistant tuberculosis in southwestern part of Ethiopia: a case control study. Annals of clinical microbiology and antimicrobials, 2018. 17(1): p. 30.

20. Hirpa, S., et al., Determinants of multidrug-resistant tuberculosis in patients who underwent first-line treatment in Addis Ababa: a case control study. BMC Public Health, 2013. 13(1): p. 782.

21. Fanta Desissa, T.W.a.T.B., Risk factors for the occurrence of multidrug-resistant tuberculosis among patients undergoing multidrug-resistant tuberculosis treatment in East Shoa,Ethiopia. 2018. 
22. Commission, F.D.R.o.E.P.C., Summary and Statistical report of the 2007 population and housing census. Addis Ababa, Ethiopia, 2008.

23. Desissa, F., T. Workineh, and T. Beyene, Risk factors for the occurrence of multidrug-resistant tuberculosis among patients undergoing multidrug-resistant tuberculosis treatment in East Shoa, Ethiopia. BMC Public Health, 2018. 18(1): p. 422.

24. Mulisa, G., et al., Multidrug-resistant Mycobacterium tuberculosis and associated risk factors in Oromia Region of Ethiopia. International Journal of Infectious Diseases, 2015. 39: p. 57-61.

25. Mulu, W., et al., Risk factors for multidrug resistant tuberculosis patients in Amhara National Regional State. African health sciences, 2015. 15(2): p. 368-377.

26. Andrews, J.R., et al., Predictors of multidrug-and extensively drug-resistant tuberculosis in a high HIV prevalence community. PloS one, 2010. 5(12): p. e15735.

27. Tadesse, F., Risk factors for multi-drug resistant tuberculosis in Addis Ababa, Ethiopia. Universal Journal of Public Health, 2015. 3(2): p. 65-70.

28. Chuchottaworn, C., et al., Risk factors for multidrug-resistant tuberculosis among patients with pulmonary tuberculosis at the Central Chest Institute of Thailand. PLoS One, 2015. 10(10): p. e0139986.

29. Fregona, G., et al., Risk factors associated with multidrug-resistant tuberculosis in Espirito Santo, Brazil. Revista de saude publica, 2017. 51: p. 41.

30. Espinal, M., et al., Determinants of drug-resistant tuberculosis: analysis of 11 countries. The International Journal of Tuberculosis and Lung Disease, 2001. 5(10): p. 887-893.

31. Asgedom, S.W., M. Teweldemedhin, and H. Gebreyesus, Prevalence of Multidrug-Resistant Tuberculosis and Associated Factors in Ethiopia: A Systematic Review. Journal of pathogens, 2018. 2018.

32. Rifat, M., et al., Factors related to previous tuberculosis treatment of patients with multidrug-resistant tuberculosis in Bangladesh. BMJ open, 2015. 5(9): p. e008273.

33. Abdella, K., et al., Drug resistance patterns of Mycobacterium tuberculosis complex and associated factors among retreatment cases around Jimma, Southwest Ethiopia. BMC Public Health, 2015. 15(1): p. 599.

34. Ricks, P.M., et al., Characteristics of multidrug-resistant tuberculosis in Namibia. BMC infectious diseases, 2012. 12(1): p. 385.

\section{Tables}

Table 1: Socio-demographic characteristics of MDR-TB in Tigray regional state, Ethiopia, $2019(n=254)$ 


\section{Variables}

Cases $(\mathrm{n}=85 \quad$ controls $(\mathrm{n}=169)$

Number Percentage Number Percentage

\section{Age}

\begin{tabular}{lllll}
$18-25$ & 23 & 27.1 & 37 & 21.9 \\
\hline $26-45$ & 44 & 51.8 & 93 & 55 \\
\hline$>45$ & 18 & 21.2 & 39 & 23.1
\end{tabular}

Sex

\begin{tabular}{lllll} 
Male & 55 & 64.7 & 99 & 58.6 \\
\hline Female & 30 & 35.3 & 70 & 41.4
\end{tabular}

Religion

\begin{tabular}{lllll} 
Orthodox & 76 & 89.4 & 157 & 92.9 \\
\hline Muslim & 9 & 10.6 & 12 & 7.1
\end{tabular}

\section{Occupation}

\begin{tabular}{lllll}
\hline Farmer & 26 & 30.6 & 49 & 29 \\
\hline Merchant & 10 & 11.8 & 32 & 18.9 \\
\hline Government employer & 7 & 8.2 & 14 & 8.3 \\
\hline Unemployed & 29 & 34.1 & 40 & 23.7 \\
\hline Others** & 13 & 15.3 & 34 & 20.1
\end{tabular}

\section{Family size}

\begin{tabular}{lllll}
\hline $1-3$ & 26 & 30.6 & 57 & 33.7 \\
\hline $4-6$ & 49 & 57.6 & 91 & 53.8 \\
\hline $7-11$ & 10 & 11.8 & 21 & 12.4
\end{tabular}

Marital status

\begin{tabular}{lllll} 
Single & 32 & 37.6 & 52 & 30.8 \\
\hline Married & 37 & 43.5 & 78 & 46.2 \\
\hline Divorced /widowed & 16 & 18.8 & 39 & 23.1
\end{tabular}

monthly income

\begin{tabular}{lllll}
\hline Up to 500 & 10 & 11.8 & 14 & 8.3 \\
\hline $501-1500$ & 48 & 56.5 & 94 & 55.6
\end{tabular}




\begin{tabular}{|c|c|c|c|c|}
\hline $1501-2000$ & 13 & 15.3 & 18 & 10.7 \\
\hline$>2001$ & 14 & 16.5 & 43 & 25.4 \\
\hline \multicolumn{5}{|c|}{ number of rooms } \\
\hline 1 & 32 & 37.6 & 70 & 41.4 \\
\hline $2-3$ & 40 & 47.1 & 83 & 49.1 \\
\hline$>=4$ & 13 & 15.3 & 16 & 9.5 \\
\hline \multicolumn{5}{|c|}{ Presence of widows } \\
\hline No & 20 & 23.5 & 40 & 23.7 \\
\hline Yes & 65 & 76.5 & 129 & 76.3 \\
\hline \multicolumn{5}{|l|}{ number of } \\
\hline \multicolumn{5}{|c|}{ windows $(\mathrm{n}=65)$} \\
\hline 1 & 30 & 46.2 & 46 & 35.7 \\
\hline $2-3$ & 29 & 44.6 & 67 & 51.9 \\
\hline$>=4$ & 6 & 9.2 & 16 & 12.4 \\
\hline \multicolumn{5}{|c|}{ open window } \\
\hline No & 9 & 13.8 & 19 & 14.7 \\
\hline Yes & 56 & 86.2 & 110 & 85.3 \\
\hline
\end{tabular}

N.B: others in occupation includes private employee, students, daily worker and pensioned

Table 2: Behavioral factors of multidrug resistant tuberculosis among adults undergoing treatment for tuberculosis in Tigray regional state, Ethiopia, 2019 ( $\mathrm{n}=254)$ 


\begin{tabular}{|c|c|c|c|c|}
\hline \multirow[t]{2}{*}{ Variables } & \multicolumn{4}{|c|}{$\begin{array}{l}\text { Cases }(n=85) \\
\text { controls }(n=169)\end{array}$} \\
\hline & Number & percentage & Number & percentage \\
\hline \multicolumn{5}{|c|}{ drinking alcohol } \\
\hline No & 67 & 78.8 & 127 & 75.1 \\
\hline Yes & 18 & 21.2 & 42 & 21.2 \\
\hline \multicolumn{5}{|c|}{ cigarette smoking } \\
\hline No & 78 & 91.8 & 152 & 89.9 \\
\hline Yes & 7 & 8.2 & 17 & 10.1 \\
\hline \multicolumn{5}{|c|}{$\begin{array}{l}\text { Lived with cigarette } \\
\text { smoker }\end{array}$} \\
\hline No & 79 & 92.9 & 149 & 88.2 \\
\hline Yes & 6 & 7.1 & 20 & 11.8 \\
\hline \multicolumn{5}{|c|}{ Imprison status } \\
\hline No & 72 & 84.7 & 148 & 87.6 \\
\hline Yes & 13 & 15.3 & 21 & 12.4 \\
\hline
\end{tabular}

Table 3: Clinical related factors of multidrug resistant tuberculosis among adults undergoing treatment for tuberculosis in Tigray regional state, Ethiopia, 2019 ( $\mathrm{n}=254)$ 


\section{Number of TB episodes}

$\begin{array}{lllll}\text { Once } & 29 & 34.1 & 120 & 71 \\ >=2 & 56 & 65.9 & 49 & 29\end{array}$

\section{Interruption first line anti TB}

treatment

$\begin{array}{lllll}\text { No } & 44 & 78.6 & 40 & 81.6 \\ \text { Yes } & 12 & 21.4 & 9 & 18.4\end{array}$

\section{Reason of interruption}

Side effects

Forgetting

Feeling better

DOT

No

Yes

Place of treatment

Health center

Hospital

Contact with known TB

No

22

34

60.7

21

42.9

22

39.3

28

57.1
Yes

I don't remember

Contact with known MDR-TB

No

Yes

I don't remember
27

15

43
25.9

27.1

47.0
62

43

64

97

57.4

17.6

50.6
4

68
36.7

25.4

37.9 


\begin{tabular}{lllll} 
Cured & 15 & 26.8 & 15 & 30.6 \\
\hline Completed & 21 & 37.5 & 25 & 51.1 \\
\hline Lost follow up & 10 & 17.9 & 8 & 16.3 \\
\hline Failure & 10 & 17.9 & 1 & 2 \\
\hline Chronic disease status & & & & \\
\hline No & 78 & 91.8 & 149 & 88.2 \\
\hline Yes & 7 & 8.2 & 20 & 11.8 \\
\hline
\end{tabular}

N.B: $\mathrm{DOT}=$ Directly Observed Therapy; $\mathrm{MDR}=$ Multidrug Resistance; $\mathrm{TB}=$ Tuberculosis; $\mathrm{Rx}=$ Treatment

Table 4: Determinant factors of multidrug-resistant tuberculosis among adults undergoing treatment for tuberculosis in Tigray regional state, Ethiopia, 2019 


\begin{tabular}{lllllll}
\hline Variables & Controls & Cases & COR & p- & AOR & P- \\
& $n(\%)$ & $n(\%)$ & & value & & value
\end{tabular}

\section{Residence}

Urban

107(63.3) 37(43.5) 1

1

Rural

$62(36.7) \quad 48(56.5) \quad 2.24(1.3-\quad 0.003$

2.58(1.4- 0.002 3.8)

4.6) *

patient category

New

120(71) 29(34.1) 1

1

Relapse

$31(18.3) \quad 34(40) \quad 4.5(2.4-\quad 0.0001$

8.5)

3.86(1.98- $\quad 0.001$

7.5) *

Return after lost follow up

$7(4.1)$

$8(9.4)$

$4.7(1.5-\quad 0.005$

5.4(1.69- $\quad 0.004$

14.1)

17) *

Treatment failure

$11(6.5)$

14(16.5)

5.3(2.1-

0.0001

$5.2(2-13) * 0.01$

12.8)

Hx of close contact with

known TB

No

62(36.7) 22(25.9) 1

1

Yes

$$
\text { 43(25.4) 23(27.1) }
$$

1.5(0.75-

0.077

1.67(0.76-

0.197

3.0)

3.6)

I don't remember

$$
\text { 64(37.9) } 40(47.1)
$$

1.57(0.78- 0.2

3.2)

\section{HIV status}

No 
N.B: * and p-value $<0.05$ shows independent variables associated with the outcome variable and" 1" indicates reference. $\mathrm{COR}=$ Crude Odds Ratio; $\mathrm{AOR}=$ Adjusted Odds Ratio; $\mathrm{Hx}=$ History

\section{Figures}

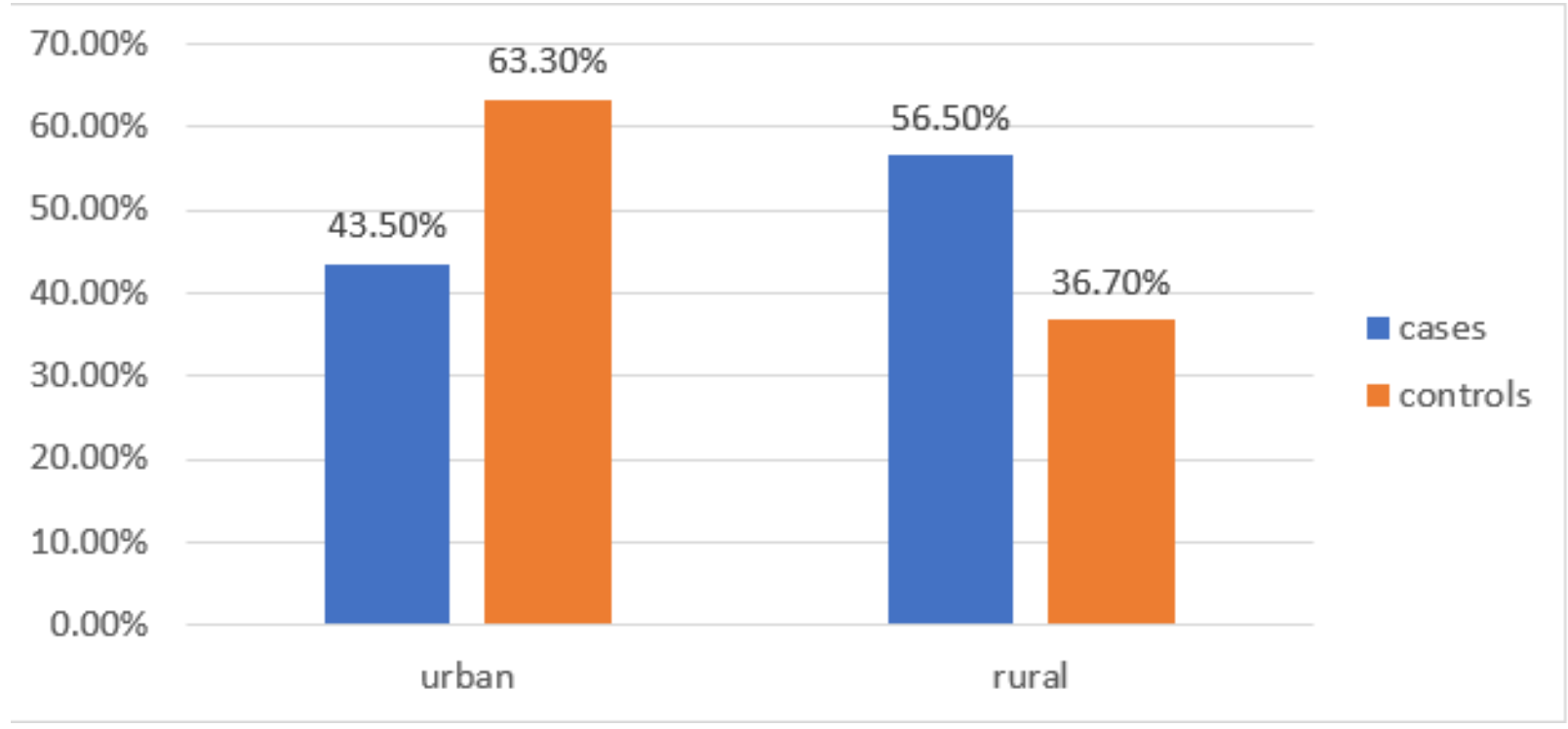

Figure 1

Distribution of MDR TB among adult residences undergoing treatment for tuberculosis in Tigray, Ethiopia, 2019

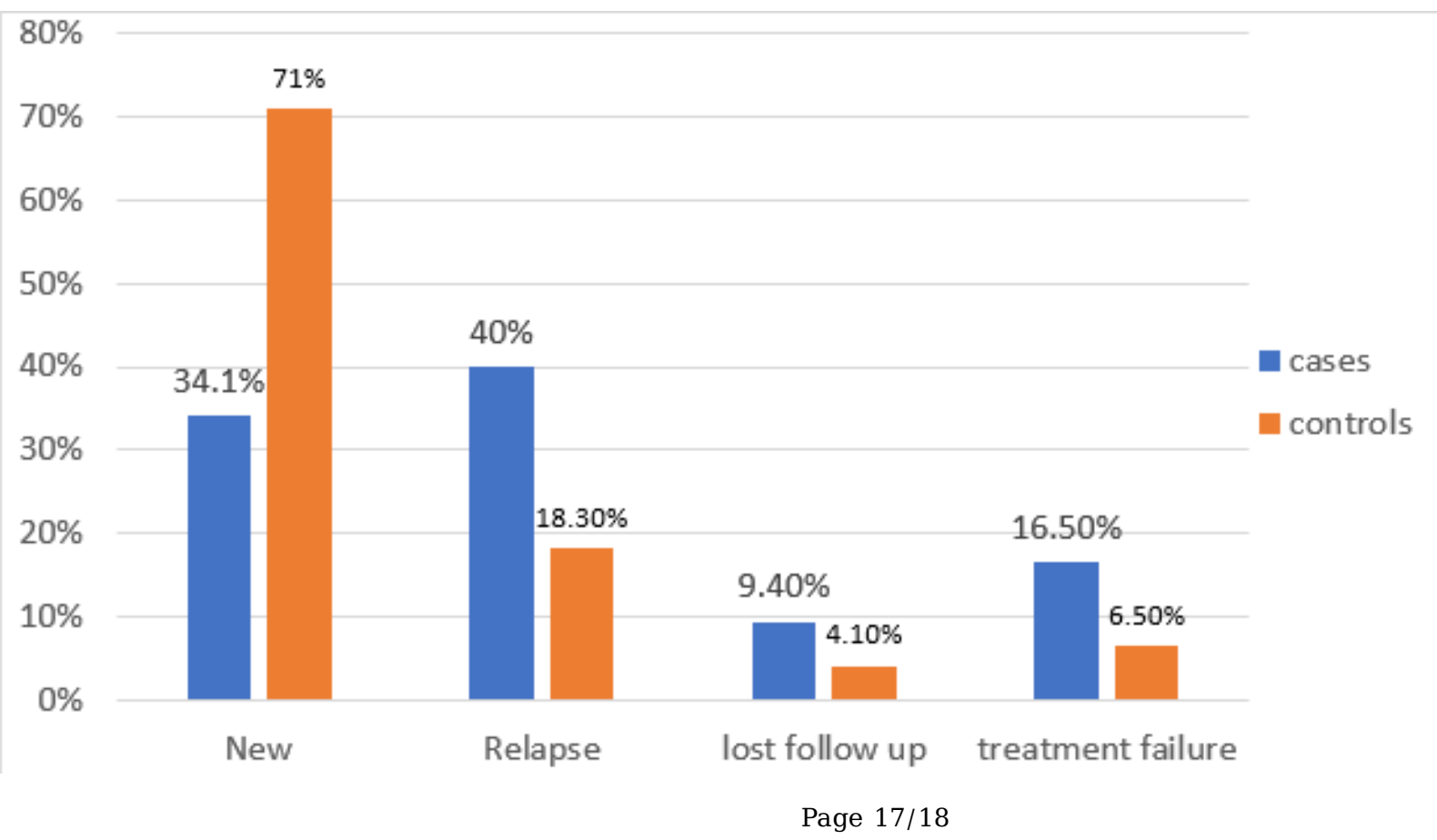


Figure 2

Treatment category of multi-drug resistance tuberculosis among adults undergoing treatment for tuberculosis in Tigray, Ethiopia, 2019 